\title{
Dynamics of Monofunctional Polybutadienyl Lithium Chains Aggregated in Benzene
}

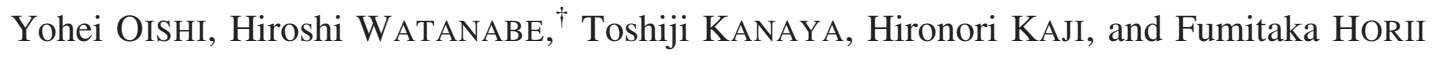 \\ Institute for Chemical Research, Kyoto University, Gokasho, Uji 611-0011, Japan
}

(Received August 24, 2005; Accepted October 29, 2005; Published March 15, 2006)

\begin{abstract}
Viscosity, ${ }^{7} \mathrm{Li}-\mathrm{NMR}$, and neutron/light scattering measurements were conducted for living monoanionic polybutadienyl lithium chains of various molecular weights $M$ and concentrations $C$. In benzene, the living chains aggregated with each other at their Li ends to form star-like tertramers as the major component, as confirmed from the viscosity and scattering measurements. An average time $\tau_{\text {dif }}$ required for the tetrameric aggregate to diffuse over a distance to a neighboring aggregate was estimated from the viscosity data with the aid of the bead-spring model (valid at the small $C$ and $M$ examined). This $\tau_{\text {dif }}$, of the order of $10^{-5}-10^{-7} \mathrm{~s}$, was much smaller than the $\mathrm{Li}-\mathrm{Li}$ exchange time $\tau_{\mathrm{ex}}$ determined from the ${ }^{7} \mathrm{Li}$-NMR measurement. The large difference between $\tau_{\mathrm{dif}}$ and $\tau_{\mathrm{ex}}$ indicates that the dissociation of the aggregates, occurring through the $\mathrm{Li}-\mathrm{Li}$ exchange, is much less frequent than the thermal collision of the aggregates. The $M, C$, and $T$ dependencies of the exchange time (dissociation time) $\tau_{\mathrm{ex}}$ were satisfactorily described by an empirical equation, $\tau_{\mathrm{ex}} \propto Q_{\mathrm{os}} \tau_{\mathrm{dif}} \exp (\Delta E / R T)$ where $R$ and $T$ were the gas constant and absolute temperature, respectively, and $Q_{\mathrm{os}}$ denoted an osmotic barrier for mutual approach of the aggregates carrying the aggregated Li species at the center. The activation energy $\Delta E(\cong 88 \mathrm{~kJ} / \mathrm{mol})$ was considerably smaller than the bare energy required for breaking the $\mathrm{Li}-\mathrm{Li}$ bonds and releasing isolated $\mathrm{Li}$ species. These results suggest that the collision of the aggregates (under the osmotic barrier) is required for the dissociation of the aggregates and the dissociation results from a cooperative exchange of Li species occurring through formation of a larger, transient aggregate.

[DOI 10.1295/polymj.38.277]

KEY WORDS Living Monoanionic Chain / Aggregates / Dynamics /
\end{abstract}

It is well known that living anionic polymer chains synthesized with organolithium (RLi) initiators aggregate with each other through their Li-ends in nonpolar solvents. ${ }^{1-19}$ For monoanionic chains such as polystyrenyllithium (PSLi) and polybutadienyllithium (PBLi) polymerized with butyllithium, the analysis of polymerization kinetics ${ }^{1-7,11}$ and the vacuum viscosmetry ${ }^{1,11-15}$ suggested the aggregation functionality (number of chains per aggregate) of $f=2-4$ for a main component of the aggregates. These small aggregates coexist with a minor amount of large aggregates with $f>100$, as revealed from light/neutron scattering experiments. ${ }^{8-10,16-18}$ Recently, the neutron scattering and ${ }^{1} \mathrm{H}$ NMR studies were conducted during the polymerization process of PBLi in heptane to reveal an interesting decrease of $f$ with increasing conversion (from $f$ of the order of 100 to $f \cong 4$ for the main component of the aggregates). This decrease was intimately related to the NMR-determined initiation/polymerization rates.

Thus, the static structure of the aggregates of the living monoanionic chains in nonpolar solvents has been elucidated to a considerable depth. However, the lifetime and dissociation kinetics of the aggregates were much less clearly understood, partly because the dissociation is slow and most of the dynamic process- es are governed by the thermal motion of the small aggregates that occurs prior to the dissociation. For this problem, we recently focused on living bianionic polybutadienyl dilithium ( $\mathrm{PBLi}_{2}$ ) chains: ${ }^{19}$ A dynamic network is formed through the association of $\mathrm{Li}$ at both ends of these chains, and its viscoelastic relaxation occurs through the thermal dissociation of the associated Li domain (because the motion of the undissociated network is very slow). For the $\mathrm{PBLi}_{2}$ chains with the molecular weight $=14 \times 10^{3}$ and the concentration $=2.2 \mathrm{wt} \%$, the viscoelastic experiment was successfully conducted in vacuum to determine the relaxation time $\tau$ (being of the order of $0.1 \mathrm{~s}$ at room temperature). This $\tau$ was close to a time required for an exchange of $\mathrm{Li}$ species (i.e., the time required for dissociation of the $\mathrm{Li}$ domain) determined from the ${ }^{7} \mathrm{Li}-\mathrm{NMR}$ measurements, indicating that the aggregates indeed had a very long lifetime.

The above result demonstrated that the viscoelastic and ${ }^{7} \mathrm{Li}$-NMR methods are very useful for studying the slow kinetics of the aggregated bianionic chains. For solutions of the monoanionic PBLi chains, the relaxation time reflecting the thermal motion of the aggregates is too short to be detected viscoelastically (unless the chain molecular weight $M$ is intractably large). However, we can still measure the viscosity

${ }^{\dagger}$ To whom correspondence should be addressed (Tel/Fax: +81-774-38-3135, E-mail: hiroshi@scl.kyoto-u.ac.jp). 
of such monoanionic chains, and a combination of the viscosity and ${ }^{7} \mathrm{Li}$-NMR measurements is expected to be very useful for studying the dissociation kinetics of the monoanionic chains. Thus, we conducted these measurements as well as the light/neutron scattering measurements to characterize the dynamic behavior of the aggregates of low- $M$ PBLi chains having various concentrations $C$ in deuterated benzene. The NMR-determined $\mathrm{Li}-\mathrm{Li}$ exchange time (lifetime of the aggregates) increased with decreasing $C$ and increasing $M$, suggesting that the dissociation requires the thermal collision of the aggregates under an osmotic barrier and that the dissociation occurs through a cooperative exchange of Li species. Details of these results are presented in this paper.

\section{EXPERIMENTAL}

\section{Materials}

Protonated polybutadienyl lithium (PBLi) monoanionic living chains were synthesized in deuterated benzene $(\mathrm{d}-\mathrm{Bz})$ at room temperature with high vacuum techniques using glass flasks/ampoules, breakable seals, constrictions, and teflon-coated magnetic bars. The initiator, $s$-butyllithium, was synthesized from lithium metal and $s$-butylbromide in heptane. All chemical reagents (purchased from Aldrich Co. and Wako Co.) were purified with the standard methods. ${ }^{1}$ The microstructure of the PBLi chains synthesized in the above condition is cis:trans:vinyl $\cong 40: 50: 10{ }^{1}$

Three PBLi samples were polymerized at a concentration $\cong 10 \mathrm{wt} \%$. The reactor $(100 \mathrm{~mL}$ glass flask) containing the $\mathrm{d}-\mathrm{Bz}$ solution of each PBLi sample was connected, at its breakable seal, to a splitting apparatus carrying a glass capillary viscometer with a solvent reservoir, NMR tubes, a glass ampoule containing a separately prepared $\mathrm{Bz}$ solution of hexyldiphenylethylenyl lithium (DPELi) anion, and an ampoule containing degassed methanol. After thorough evacuation, this apparatus was sealed off and its inner wall was rinsed with the DPELi solution. Then, this solution was recovered in its original ampoule and sealed off, the breakable seal of the reactor was opened, and a fraction of the $\mathrm{PBLi} / \mathrm{d}-\mathrm{Bz}$ solution therein was split into the viscometer and NMR tubes. Then, a fraction of $\mathrm{d}-\mathrm{Bz}$ remaining in the reactor was vacuum-distilled into the viscometer and NMR tubes to prepare the test PBLi solutions of various concentrations. For one sample (with $M=5400$ ), the solution was also split into neutron and light scattering cells made of quartz and glass, respectively.

After sealing off the viscometer, NMR tubes, and the scattering cells, the PBLi/d-Bz solution remaining in the reactor was deactivated with the degassed methanol. This deactivated solution was poured into a large
Table I. Characteristics of PB samples

\begin{tabular}{lccc}
\hline Code & $10^{-3} M^{\mathrm{a}}$ & $M_{\mathrm{w}} / M_{\mathrm{n}}$ & $C^{* \mathrm{~b}} / \mathrm{g} \mathrm{cm}^{-3}$ \\
\hline PB-3 & 2.7 & 1.02 & 0.14 \\
PB-5 & 5.4 & 1.02 & 0.099 \\
PB-10 & 9.6 & 1.03 & 0.074 \\
\hline
\end{tabular}

${ }^{\mathrm{a}}$ weight-average molecular weight. ${ }^{\mathrm{b}}$ overlapping concentration for deactivated chains.

excess of methanol containing $\cong 0.005 \mathrm{wt} \%$ of antioxidant, butylhydroxy toluene (BHT). The deactivated PB samples precipitated in methanol were thoroughly dried in vacuum and recovered.

These deactivated samples were characterized with GPC (CO-8020 and DP-8020, Tosoh). The eluent was THF, and monodisperse linear PB standards ${ }^{20}$ were utilized to calibrate the elution volume. The weightaverage molecular weights and the polydispersity indices determined from this calibration are summarized in Table I. The sample code numbers indicate the molecular weight in unit of 1000 .

\section{Measurements}

Viscosity. The sealed capillary viscometers containing the monoanionic $\mathrm{PBLi} / \mathrm{d}-\mathrm{Bz}$ solutions were soaked in a temperature-controlled water bath and the flow time through the capillary was measured. (The viscoelastic relaxation of the solutions was too fast to be detected with the previously used magnetically floating ball rheometer. ${ }^{19}$ ) After the measurement at several temperatures $T\left(=10-34^{\circ} \mathrm{C}\right)$, the viscometer was opened to recover the $\mathrm{PB} / \mathrm{d}-\mathrm{Bz}$ solutions. The $\mathrm{PB}$ concentration $C$ (in mass/volume unit) was determined by measuring the volume of the solutions and the weight of the PB chains therein (recovered after full evaporation of d-BZ).

After the above operation, the capillary viscometer was thoroughly washed with protonated benzene (h$\mathrm{Bz}$ ) and dried. Then, the capillary flow time was measured for two solvents, $\mathrm{h}-\mathrm{Bz}$ and d-Bz. The viscosities of $\mathrm{d}-\mathrm{Bz}$ and the $\mathrm{PBLi} / \mathrm{d}-\mathrm{Bz}$ solutions were determined from their flow times and the viscosity of h-Bz (known as a function of temperature ${ }^{21}$ ) after a correction of the difference between the densities of $\mathrm{h}-\mathrm{Bz}$ and $\mathrm{d}-\mathrm{Bz}, \rho_{\mathrm{d}-\mathrm{Bz}}=0.95 \mathrm{~g} \mathrm{~cm}^{-3}$ and $\rho_{\mathrm{h}-\mathrm{Bz}}=$ $0.88 \mathrm{~g} \mathrm{~cm}^{-3}$.

For the deactivated $\mathrm{PB}$ samples directly recovered from the reactor, $\mathrm{d}-\mathrm{Bz}$ solutions were prepared at the concentrations $C$ identical to those of the living $\mathrm{PBLi} / \mathrm{d}-\mathrm{Bz}$ solutions. The viscosity of these deactivated $\mathrm{PB} / \mathrm{d}-\mathrm{Bz}$ solutions was also determined with the capillary viscometer.

NMR. For the living PBLi/d-Bz solutions sealed in the NMR tubes, ${ }^{7} \mathrm{Li}-\mathrm{NMR}$ measurements were conducted at $T=5-70^{\circ} \mathrm{C}$ with a JEOL JNM-AL400 
spectrometer operated under a static magnetic field of 9.4 T. The resonance frequency was $153.86 \mathrm{MHz}$. The measured ${ }^{7} \mathrm{Li}$ chemical shifts were expressed as the values relative to a $0.2 \mathrm{M} \mathrm{LiCl}$ solution in $\mathrm{D}_{2} \mathrm{O}$. The ${ }^{7} \mathrm{Li}$ spectra (in $\mathrm{d}-\mathrm{Bz}$ ) with and without the ${ }^{1} \mathrm{H}$-decoupling $^{22,23}$ were indistinguishable, indicating a fairly wide spatial separation between ${ }^{7} \mathrm{Li}$ and ${ }^{1} \mathrm{H}$ (in the PB backbone). No ${ }^{1} \mathrm{H}$-decoupling was applied to the ${ }^{7} \mathrm{Li}$ spectra reported in this paper.

After the measurement, the NMR tubes were opened to recover the $\mathrm{PB} / \mathrm{d}-\mathrm{Bz}$ solutions. The $\mathrm{PB}$ concentration $C$ (in mass/volume unit) was determined from the weights of the solutions and PB therein with the aid of the known densities, $\rho_{\mathrm{d}-\mathrm{Bz}}=0.95$ $\mathrm{g} \mathrm{cm}^{-3}$ and $\rho_{\mathrm{PB}}=0.92 \mathrm{~g} \mathrm{~cm}^{-3}$.

SANS and LS. Recent scattering experiments ${ }^{16-18}$ revealed that living PBLi chains form aggregates in heptane and cyclohexane and the aggregation number $f$ has some distribution: The main component of the aggregates is the tetramer $(f=4)$ but a small amount of large aggregates $(f>100)$ is also formed. Similar aggregation behavior was expected also in our PBLi/ d-Bz solutions.

To confirm this expectation, the living solution of the PB-5 sample $(M=5400)$ sealed in the scattering cell made of quartz (with the path length $=2 \mathrm{~mm}$ ) was subjected to the small angle neutron scattering (SANS) measurement at $25^{\circ} \mathrm{C}$. The measurement was made with the SANS-U spectrometer at the Neutron Scattering Laboratory, Institute for Solid State Physics, University of Tokyo (Tokai, Ibaragi). The incident neutron wavelength and its spread were $\lambda=0.7 \mathrm{~nm}$ and $\Delta \lambda / \lambda=0.1$, respectively, the beam diameter was $0.3 \mathrm{~cm}$, and the sample-to-detector distance was chosen to be 2.0 and $8.0 \mathrm{~m}$. The scattering intensity was measured as a function of the scattering vector $\mathbf{q}$, where $q=|\mathbf{q}|=[4 \pi / \lambda] \sin (\theta / 2)$ with $\theta$ being the scattering angle.

After the SANS measurement, the living PB-5 concentration $\left(C=0.023 \mathrm{~g} \mathrm{~cm}^{-3}\right)$ was determined from the weights of the solution and PB5 therein, as done after the ${ }^{7} \mathrm{Li}-\mathrm{NMR}$ measurement. A d-Bz solution of the deactivated PB-5 sample (directly recovered from the reactor) was prepared at the same $C$, and this solution was also subjected to the SANS measurement.

The SANS profiles of the living and deactivated PB-5 solutions were azimuthally symmetric. Thus, the scattering intensity was circularly averaged after subtraction of the background scattering measured for pure d-Bz. In this paper, we present these circularly averaged intensities $I(q)$ without a correction for the incoherent scattering.

For the living PB-5 solution, the static light scattering (LS) measurement was also conducted with a spectrometer (Malvern System-4700; $\lambda=488 \mathrm{~nm}$ ) to examine the existence of a small amount of large aggregates (known to be formed in heptane ${ }^{16,17}$ and cyclohexane $\left.{ }^{18}\right)$. The LS intensity $I(q)$ was measured as a function of the scattering vector $q$ and compared with the SANS intensity.

\section{RESULTS AND DISCUSSION}

\section{Viscosity Data}

For the solutions of the living and deactivated PB chains, the viscosity of $\mathrm{PB}$ chains therein, $\eta_{\mathrm{PB}}$, was evaluated by subtracting the solvent (d-Bz) viscosity from the solution viscosity. The temperature dependence of $\eta_{\mathrm{PB}}$ is shown in Figure 1. We first note that $\eta_{\mathrm{PB}}$ is larger for the living PB chain (unfilled symbols)

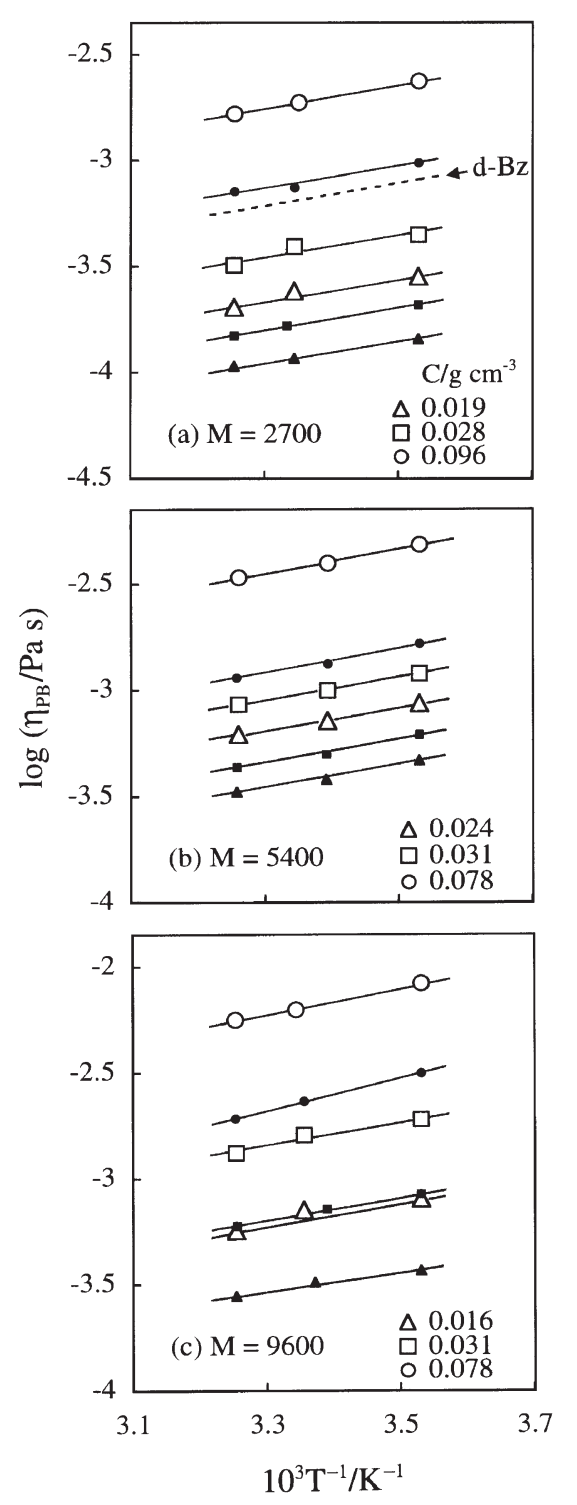

Figure 1. Temperature dependence of the viscosities of the living and deactivated PB chains (large and small symbols) with $M$ and $C$ as indicated. $C$ is the same for the living and deactivated chains. The dotted line in the top panel denotes the viscosity of the solvent (d-PB). 


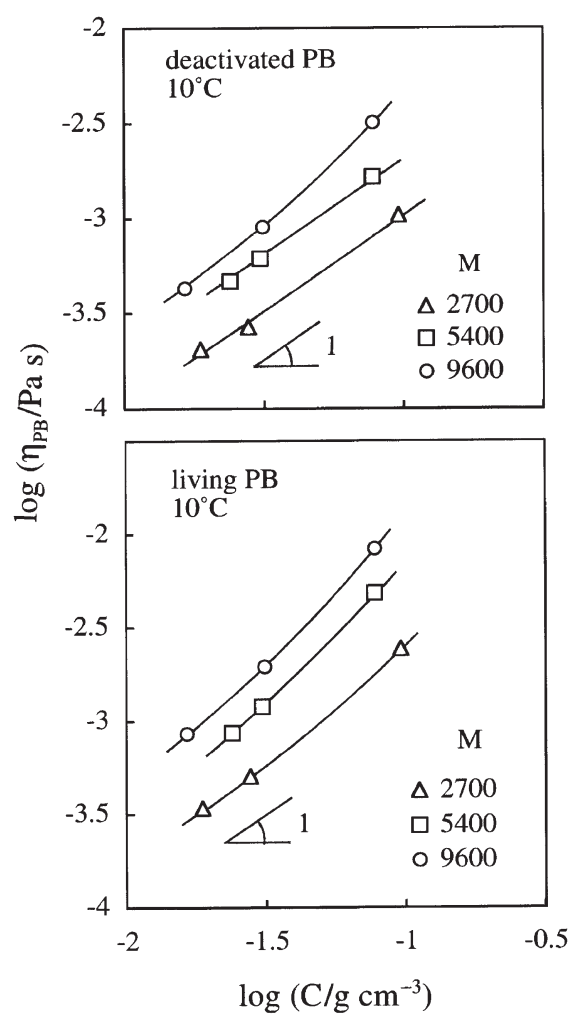

Figure 2. Concentration dependence of the viscosities at $10^{\circ} \mathrm{C}$ obtained for the deactivated and living PB chains with $M$ as indicated (top and bottom panels).

than for the deactivated chain (small filled symbols) having the same $C$. This result unequivocally indicates that the living chains form the aggregates to enlarge their viscosity at the given $C$ (due to an increase of their relaxation time), as pointed out also in the previous work. ${ }^{1,11-15} \mathrm{We}$ also note that both of the living and deactivated chains exhibit the Arrhenius behavior, $\eta_{\mathrm{PB}} \propto \exp \left(\Delta E_{\eta} / R T\right)$ with $R=$ gas constant and $T=$ absolute temperature. The activation energy $\Delta E_{\eta}$ $\left(\cong 9.7 \mathrm{~kJ} \mathrm{~mol}^{-1}\right)$ is similar for these chains and close to that for pure $\mathrm{d}-\mathrm{Bz}$ ( $c f$. dotted line in the top panel).

In Figure 2, the $\eta_{\mathrm{PB}}$ data at a constant temperature $\left(10^{\circ} \mathrm{C}\right)$ are plotted against $C$. For the deactivated PB chains (top panel), $\eta_{\mathrm{PB}}$ is almost proportional to $C$ except at the highest $C$ examined for PB-10 ( $M=$ 9600). Similar behavior was noted at different temperatures. This $C$ dependence of $\eta_{\mathrm{PB}}$ can be related to the overlapping concentration $C^{*}$ of the PB chains. Since the chains have rather small $M$, we may safely neglect the excluded volume effect and estimate $C^{*}$ from the unperturbed radius of gyration $R_{\mathrm{g}, \theta}$ as

$$
C^{*}=\frac{\left\{M / N_{\mathrm{A}}\right\}}{\left\{4 \pi R_{\mathrm{g}, \theta}^{3} / 3\right\}}
$$

where $N_{\mathrm{A}}$ is the Avogadro number. $R_{\mathrm{g}, \theta}$ is estimated from an empirical equation ${ }^{24}$

$$
R_{\mathrm{g}, \theta}=3.79 \times 10^{-2} M^{1 / 2} \mathrm{~nm}
$$

The $C^{*}$ values are summarized in Table I. The $C$ examined for the PB chains are considerably smaller than $C^{*}$, except for the highest $C\left(=0.078 \mathrm{~g} \mathrm{~cm}^{-3}\right)$ for the PB-10 chain. The viscosity is known to be essentially proportional to $C$ for such moderately dilute chains. ${ }^{25}$ (These chains exhibit no entanglement effect at all.)

The $C$ dependence of $\eta_{\mathrm{PB}}$ of the living PB chains is stronger than that of the deactivated chains and the proportionality is observed only for the shortest PB-3 chain $(M=2700)$ at $C<0.03 \mathrm{~g} \mathrm{~cm}^{-3}$; see the bottom panel of Figure 2. This strong $C$ dependence provides us with an insight about the aggregated structure: For the $f$-arm star-like aggregates in the absence of the excluded volume effect, the overlapping concentration $C_{\mathrm{agg}}{ }^{*}$ can be related to $C^{*}$ of the deactivated chain as

$$
C_{\text {agg }}{ }^{*}=f^{5 / 2}(3 f-2)^{-3 / 2} C^{*}
$$

(This relationship is derived from a ratio of $R_{\mathrm{g}, \theta}$ 's of $f$-arm star and linear chains having the same $M$, $R_{\mathrm{g}, \theta}(\operatorname{star}) / R_{\mathrm{g}, \theta}($ linear $\left.)=(3 f-2)^{1 / 2} / f .^{26}\right)$ The minimum values of $C_{\mathrm{agg}}{ }^{*}$ in the range of $f \geq 3,0.083$ and $0.12 \mathrm{~g} \mathrm{~cm}^{-3}$ for the PB-5 and PB-3 chains ( $M=$ 5400 and 2700), are still larger than the highest $C$ examined for these chains. Thus, if $f$ does not change with $C$, the star-like aggregates at those $C$ should behave as moderately dilute, non-entangled star chains to exhibit the proportionality between $\eta_{\mathrm{PB}}$ and $C$. Indeed, the scattering experiments conducted for similar PBLi solutions ${ }^{18}$ suggest that $f$ does not significantly change in the range of $C$ examined here. In addition, the viscosity at a given $C$, deduced from the bead-spring (Rouse-Ham) model, ${ }^{26}$ is proportional to $4-3 f^{-1}$ and changes with $f$ only a little in the range of $f \geq 3$. Considering these points, we may conclude that the strong $C$ dependence of $\eta_{\mathrm{PB}}$ seen for the living $\mathrm{PBLi} / \mathrm{d}-\mathrm{Bz}$ solutions is mainly attributable to the coexisting large aggregates. Indeed, the existence of such large aggregates was confirmed from the scattering measurement, as explained later in more details.

Figure 3 shows the dependence of $\eta_{\mathrm{PB}}$ at $10^{\circ} \mathrm{C}$ and at fixed $C\left(=0.024\right.$ and $\left.0.078 \mathrm{~g} \mathrm{~cm}^{-3}\right)$ on the molecular weight $M$ of the PB chain (= arm in the aggregates). These $C$ values are the lowest and highest values examined for the PB-5 chain, and $\eta_{\mathrm{PB}}$ of the PB-3 and PB-10 chains at those $C$ were evaluated from short interpolation of the data shown in Figure 2.

The $\eta_{\mathrm{PB}}$ of the deactivated chains at $C=0.078$ $\mathrm{g} \mathrm{cm}^{-3}$ is proportional to $M$, and the chains essentially behave as the Rouse chains at this $C$; see top panel of Figure 3. As $C$ is decreased to $0.024 \mathrm{~g} \mathrm{~cm}^{-3}$, the $M$ dependence becomes a little weaker but this proportionality still holds in an approximate sense. (Note that the dependence at $C=0.024 \mathrm{~g} \mathrm{~cm}^{-3}$ is much 


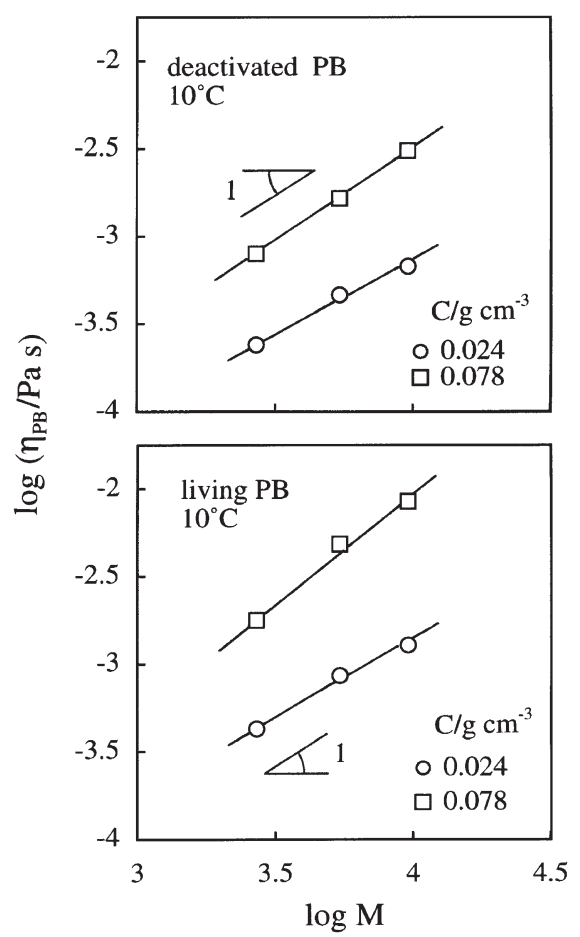

Figure 3. Molecular weight dependence of the viscosities of the deactivated and living PB chains at $10^{\circ} \mathrm{C}$ with $C$ as indicated (top and bottom panels).

stronger than that expected for the Zimm chain, ${ }^{26}$ $\eta_{\mathrm{PB}} \propto M^{1 / 2}$.) Similar results were observed at all temperatures examined. The Rouse-like $\eta_{\mathrm{PB}}$ data of the deactivated chains are later utilized in the analysis of kinetics of the exchange of $\mathrm{Li}$ species.

The $M$ dependence of $\eta_{\mathrm{PB}}$ of the living PB chains is stronger than that of the deactivated chains; $c f$. bottom panel of Figure 3. As noted from the above argument for $C_{\text {agg }}{ }^{*}$, the small star-like aggregates should behave as the moderately dilute chains to exhibit the RouseHam behavior. Thus, the strong $M$ dependence seen for the living chains may be again related to a small amount of the large aggregates that coexists with the star-like aggregates and would have a non-negligible contribution to $\eta_{\mathrm{PB}}$. Similar results were found at all temperatures examined.

\section{Scattering Data}

For the living and deactivated PB-5 chains $(M=$ 5400) at a concentration $C=0.023 \mathrm{~g} \mathrm{~cm}^{-3}$ (PB volume fraction $\phi=0.025$ ), Figure 4 shows doublelogarithmic plots of the scattering intensity $I(q)$ at $25^{\circ} \mathrm{C}$ against the wave vector $q$. The data at high $q$ $\left(\geq 0.1 \mathrm{~nm}^{-1}\right)$ are the SANS intensities of the living and deactivated chains, and the data at low $q$ $\left(\leq 0.02 \mathrm{~nm}^{-1}\right)$ are the LS intensity of the living chain. The LS intensity data are shifted vertically in the double-logarithmic scale in a way that they smoothly merge into the high- $q$ plateau of the SANS intensity.

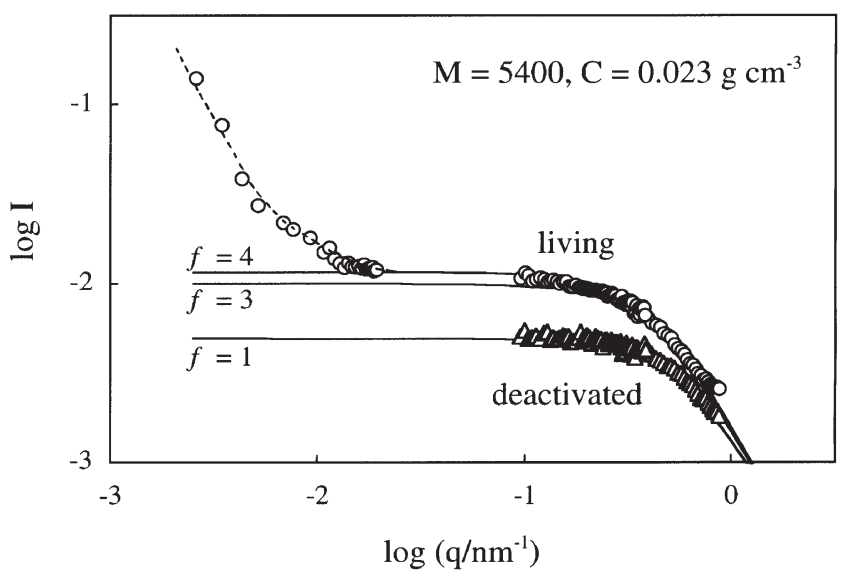

Figure 4. Scattering profiles at $25^{\circ} \mathrm{C}$ measured for the living and deactivated PB chains with $M=5400$ and $C=0.023 \mathrm{~g} \mathrm{~cm}^{-3}$. The data at high- $q$ and low- $q$ regimes were obtained from SANS and LS measurements, respectively. The solid curves indicate the profiles expected for unimer, trimer, and tetramer of the PB chains.

The difference between the SANS intensities of the living and deactivated chains at $q \geq 0.1 \mathrm{~nm}^{-1}$ indicates that most of the living chains form star-like aggregates, as explained later in more details. In addition, the strong increase of the LS intensity of the living chains in the low- $q$ regime $\left(q<0.01 \mathrm{~nm}^{-1}\right)$ demonstrates the existence of a small amount of large aggregates, as well known for living chains in nonpolar solvents. ${ }^{8-10,16-18}$ These large aggregates should have existed in all living PB solutions examined.

Stellbrink and coworkers ${ }^{9,10}$ utilized the form factor for a fractal object ${ }^{27}$ to discuss the structure of the large aggregates. However, the dissociation kinetics of the associated Li species targeted in this paper should be related to the dynamics of the main component in the aggregates (small star-like aggregates), and the large aggregates (minor component) should have negligible effect on this kinetics. For this reason, we do not attempt to analyze the $I(q)$ data on the basis of the fractal form factor. Instead, we here estimate the aggregation number $f$ for the main component that is needed in our analysis of the dissociation kinetics.

The aggregates of living anionic chains have a distribution of $f$, as demonstrated by Matsuda et al. ${ }^{18}$ However, we may still estimate $f$ for the main component of our living PB aggregates by fitting the $I(q)$ data (Figure 4) with that expected for monodisperse $f$-mer aggregates ( $f$-arm star chains). In the absence of the excluded volume effect (for low- $M$ chains), the scattering intensity of the $f$-mer aggregates is described by ${ }^{9,10}$

$$
I(q)=K \frac{\phi(1-\phi)}{\left\{\frac{1}{V_{\mathrm{m}} P(q)}+2 A_{2} \phi\right\}}
$$


with the form factor $P(q)$ being written as ${ }^{28}$

$$
\begin{aligned}
P(q)= & \frac{2}{f q^{4} R_{\mathrm{g}, \mathrm{arm}}^{4}}\left[q^{2} R_{\mathrm{g}, \mathrm{arm}}^{2}-1+\exp \left(-q^{2} R_{\mathrm{g}, \mathrm{arm}}^{2}\right)\right. \\
& \left.+\frac{f-1}{2}\left\{1-\exp \left(-q^{2} R_{\mathrm{g}, \mathrm{arm}}^{2}\right)\right\}^{2}\right]
\end{aligned}
$$

Here, $R_{\mathrm{g}, \text { arm }}$ is the unperturbed radius of gyration of the arm, $V_{\mathrm{m}}$ and $\phi$ are the molar volume and volume fraction of the star chain, $A_{2}$ is the second virial coefficient, and $K$ is a constant determined by a scattering contrast between the chain and solvent. The scattering intensity for the linear chains (dissociated arms) is given by eqs 4 and 5 with $f=1$.

The aggregation number $f$ for the main component in the aggregates can be estimated by fitting the $I(q)$ data (Figure 4) with eqs 4 and 5, but this fitting requires the $A_{2}$ value. Unfortunately, no literature data of $A_{2}$ in d-Bz are available for low- $M$ PB chains similar to our PB-5 chain $(M=5400$ and $\phi=0.025)$. Thus, we considered the $A_{2}$ data of low- $M$ polystyrenes (PS) in $\mathrm{Bz}^{9,29}$ to estimate $A_{2, \mathrm{da}} \cong 0.002$ $\mathrm{cm}^{3} \mathrm{~mol} \mathrm{~g}^{-2}$ for our deactivated PB-5 chain. (Bz is an equally good solvent for PS and PB.) For the star-like $f$-mer aggregates of the living PB-5 chains, we estimated the $A_{2 \text {,liv }}$ value from a relationship between $A_{2}$ values of the star and linear chains, ${ }^{10} A_{2, \text { liv }} \cong$ $(1.1-0.04 f) f^{-0.23} A_{2, \text { da }}$ where the factor $f^{-0.23} A_{2, \mathrm{da}}$ gives the $A_{2}$ value for a linear chain having the molecular weight identical to that of the star chain (aggregate). In Figure 4, the $I(q)$ calculated from eqs 4 and 5 with these $A_{2}$ values and $R_{\mathrm{g}, \mathrm{arm}}=3.0 \mathrm{~nm}$ are shown with the solid curves. The contrast factor $K$, common for the living and deactivated chains, was determined in a way that the calculated curve for $f=1$ agreed with the $I(q)$ data for the latter (triangles). The calculated curves for $f=3$ and 4 are close to the data for the living PB-5 chain (circles), with some preference for $f=4$. From this result as well as the results of detailed structural analysis for PBLi chains in heptane $^{16,17}$ and cyclohexane ${ }^{18}$ (suggesting $f=4$ ), we may conclude that the main component of the aggregates in our $\mathrm{PBLi} / \mathrm{d}-\mathrm{Bz}$ solutions is the tetrameric aggregate. The kinetics of the exchange of $\mathrm{Li}$ species (the dissociation kinetics) is later analyzed in relation to diffusion of the tetrameric aggregate.

\section{${ }^{7}$ Li-NMR Data}

Figures 5-7 show the ${ }^{7} \mathrm{Li}-\mathrm{NMR}$ spectra (solid curves) obtained for the PBLi/d-Bz solutions with $M$ and $C$ as indicated. The resonance frequency is $153.86 \mathrm{MHz}$, and the chemical shift $\delta$ (in $\mathrm{Hz}$ ) is expressed as the values relative to a $0.2 \mathrm{M} \mathrm{LiCl}$ solution in $\mathrm{D}_{2} \mathrm{O}$. The spectra exhibit a triple peak at low $T$, and this triple peak merges into single peak with increas-
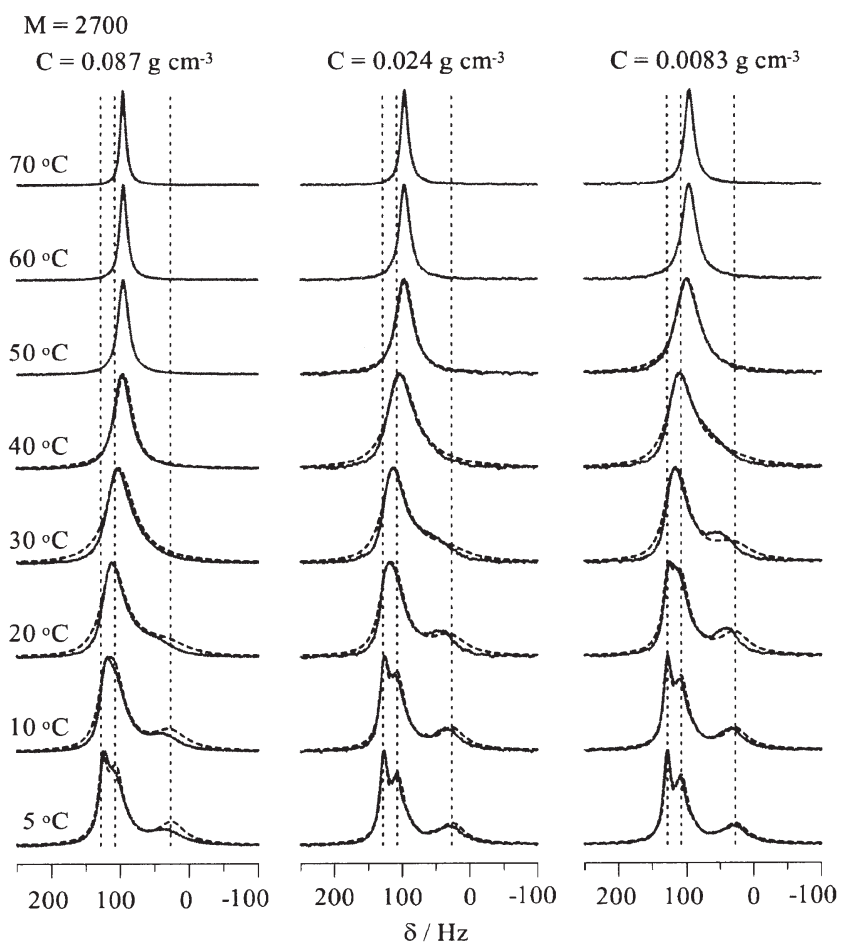

Figure 5. ${ }^{7} \mathrm{Li}-\mathrm{NMR}$ spectra obtained for living PB chains with $M=2700$ and at $C$ and $T$ as indicated (solid curves). The thick dotted curves indicate the fitted spectra calculated for exchange of four Li species.
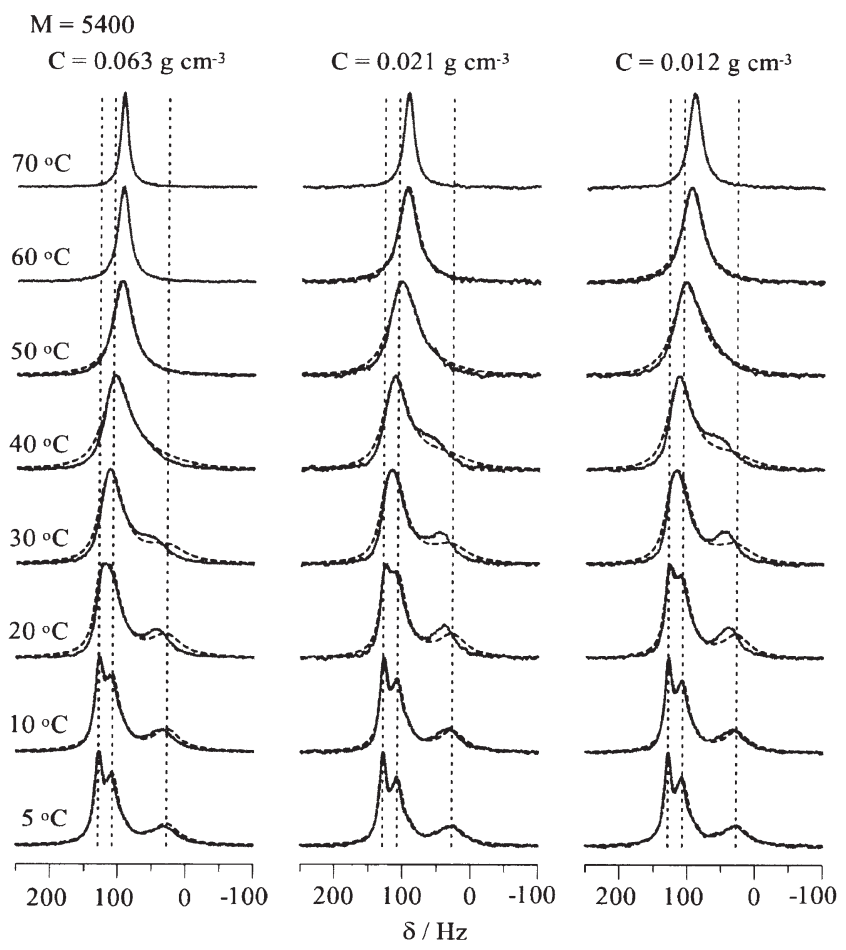

Figure 6. ${ }^{7} \mathrm{Li}-\mathrm{NMR}$ spectra obtained for living PB chains with $M=5400$ and at $C$ and $T$ as indicated (solid curves). The thick dotted curves indicate the fitted spectra calculated for exchange of four Li species. 

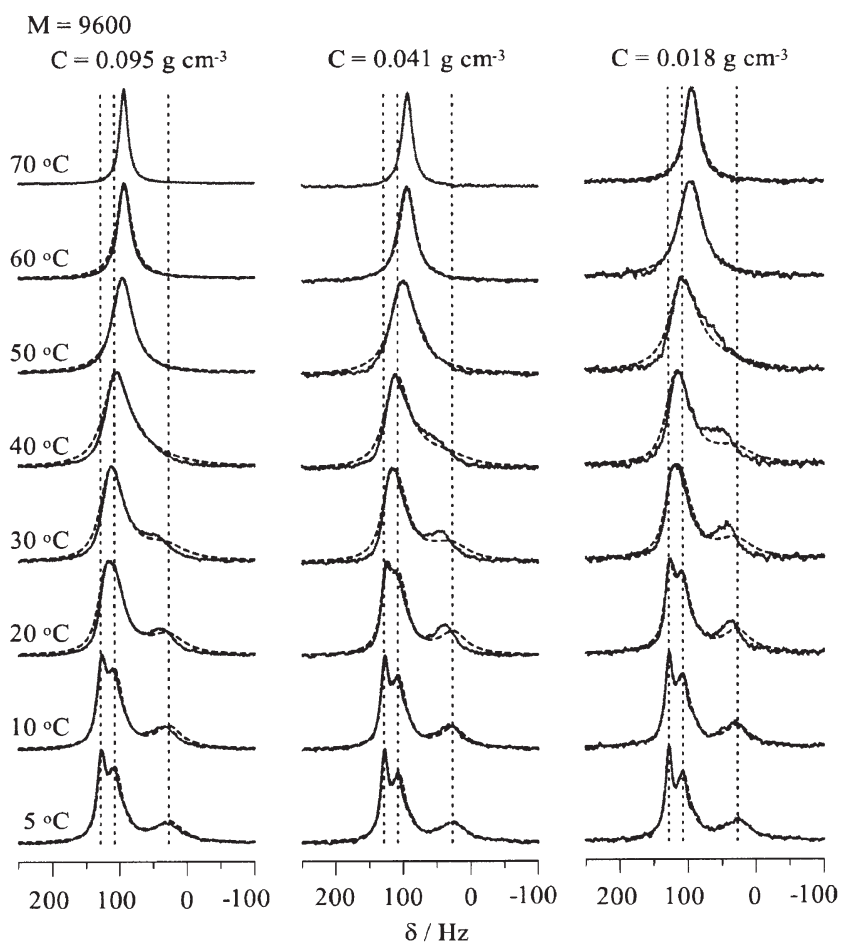

Figure 7. ${ }^{7} \mathrm{Li}-\mathrm{NMR}$ spectra obtained for living PB chains with $M=9600$ and at $C$ and $T$ as indicated (solid curves). The thick dotted curves indicate the fitted spectra calculated for exchange of four Li species.

ing $T$. From the existence of the triple peak, we can conclude that $\mathrm{Li}$ of the PBLi chains is in several different aggregation states and the exchange of these $\mathrm{Li}$ species at low $T$ is slow compared to the NMR time scale, $1 / \Delta \delta \cong 0.01 \mathrm{~s}$ with $\Delta \delta(\cong 100 \mathrm{~Hz})$ being the differences of the chemical shifts. The exchange time scale becomes comparable to/shorter than the NMR time scale on the increase of $T$ up to $70^{\circ} \mathrm{C}$, as clearly noted from the change from the triple peak to the single peak. We also note that the shape of the NMR spectra is considerably different for the monoanionic PBLi and bianionic $\mathrm{PBLi}_{2}$ chains examined in this and previous ${ }^{19}$ studies and thus the Li species for these chains are in different chemical states.

For all PBLi solutions examined, the triple peak spectrum at low $T$ was successfully deconvoluted into

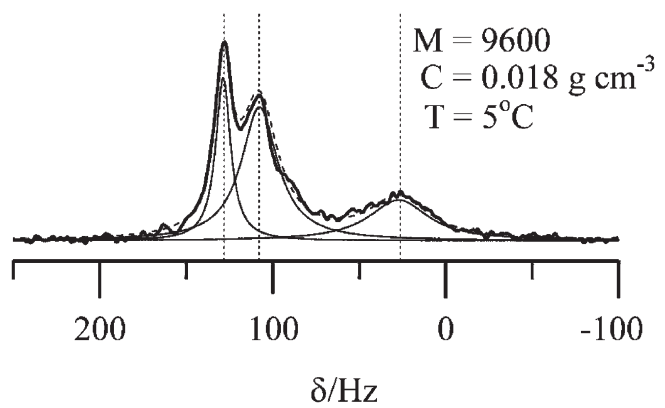

Figure 8. Deconvolution of ${ }^{7} \mathrm{Li}-\mathrm{NMR}$ spectrum of the living PB-10 solution at $5{ }^{\circ} \mathrm{C}$ (thick solid curve) into three Lorentzian peaks (thin solid curves). A ratio of the integrated intensities of the Lorentzian peaks, $H$, is close to $H_{\text {high- } \delta}: H_{\text {middle- } \delta}: H_{\text {low- } \delta}=$ $1: 2: 1$. The sum of these peaks, shown with the thin dotted curve, well reproduces the measured spectrum.

three Lorentzian peaks. As an example, Figure 8 shows the ${ }^{7}$ Li-NMR spectrum of the living PB-10 solution at $5{ }^{\circ} \mathrm{C}$ (thick solid curve) deconvoluted into the Lorentzian peaks (thin solid curves). For all solutions, a ratio of the integrated intensities of the peaks, $H$, was close to $H_{\text {high- } \delta}: H_{\text {middle- } \delta}: H_{\text {low- } \delta}=1: 2: 1$. This result suggested that (at least) four different Li species existed for the living PB chains examined, and two of them have nearly the same resonance frequency at middle $\delta$. Since details are known for neither chemical states of these species (which was the case also for the previously studied PBLi chains ${ }^{16,17}$ ) nor the number of different species $N_{\mathrm{sp}}$, we cannot accurately determine the exchange times $\tau_{\text {ex }}$ among the associated $\mathrm{Li}$ species from the NMR spectra. However, we may still obtain a reasonable estimate of $\tau_{\text {ex }}$ under simplifying assumptions that the spectra peaks seen Figures 5-7 correspond to four different species $\left(N_{\mathrm{sp}}=4\right)$ and the exchange occurs among these species at the same rate $\kappa$. The exchange time obtained under these assumptions, $\tau_{\mathrm{ex}}=1 / \kappa$, can be interpreted as an estimate of the average lifetime of the associated Li species.

Under the above assumptions, we made the line shape analysis of the ${ }^{7} \mathrm{Li}-\mathrm{NMR}$ spectra with the standard method ${ }^{30}$ utilizing an exchange matrix:

$$
\mathbf{E}_{\mathrm{x}}=\left[\begin{array}{cccc}
-\left(N_{\mathrm{sp}}-1\right) & 1 & 1 & 1 \\
1 & -\left(N_{\mathrm{sp}}-1\right) & 1 & 1 \\
1 & 1 & -\left(N_{\mathrm{sp}}-1\right) & 1 \\
1 & 1 & 1 & -\left(N_{\mathrm{sp}}-1\right)
\end{array}\right] \text { with } N_{\mathrm{sp}}=4
$$

A matrix $\mathbf{A}(\omega)$ describing the time evolution of the magnetization vector in a time scale of $1 / \omega$ is related to this $\mathbf{E}_{\mathrm{x}}$ as $\mathbf{A}=i(\omega \mathbf{I}-\boldsymbol{\Omega})-\mathbf{E}_{\mathrm{x}}$ with $i=\sqrt{-1}$, where $\mathbf{I}$ is the unit matrix and $\boldsymbol{\Omega}$ is a diagonal matrix having the spectral line frequencies of the $\mathrm{Li}$ species $\omega_{\mathrm{j}}\left(j=1-4\right.$ for $\left.N_{\mathrm{sp}}=4\right)$ as the diagonal elements. ${ }^{30}$ Utilizing the eigenvalues $\lambda_{\mathrm{j}}(\omega)=i\left(\omega-\omega_{j}\right)+N_{\mathrm{sp}} \kappa$ of a matrix $\mathbf{B}=\mathbf{A}+\kappa \tilde{\mathbf{I}}$ (where $\tilde{\mathbf{I}}$ is the "one-matrix" 
having the elements $\tilde{\mathrm{I}}_{\mathrm{jk}}=1$ for all $j$ and $k$ ), we may express the line shape $g(\omega)$ as

$$
g(\omega)=\frac{1}{N_{\mathrm{sp}}} \frac{L(\omega)}{1-\kappa L(\omega)}
$$

with

$$
\begin{aligned}
L(\omega)= & \sum_{\mathrm{j}=1}^{N_{\mathrm{sp}}} \frac{1}{\lambda_{\mathrm{j}}(\omega)}=\sum_{\mathrm{j}=1}^{N_{\mathrm{sp}}} \frac{1}{N_{\mathrm{sp}} \kappa+i\left(\omega-\omega_{\mathrm{j}}\right)} \\
& \text { and } \quad N_{\mathrm{sp}}=4
\end{aligned}
$$

The average exchange time (= lifetime of the associated Li domain), $\tau_{\mathrm{ex}}=1 / \kappa$, can be estimated by fitting the data in Figures 5-7 with the real part of $g(\omega)$ specified by eqs 7 and 8 .

For definiteness of this fitting, we assumed that the spectral line frequencies $\omega_{\mathrm{j}}$ reflecting the chemical states of the Li species do not change with $T$ and are independent of $C$ and $M$ of the PBLi chains. (The fitting becomes rather arbitrary if we allow changes of $\omega_{\mathrm{j}}$ with $T, C$, and $M$, although these changes may have occurred to some extent in the actual PBLi solutions.) In Figures 5-7, vertical dotted lines indicate the frequency shifts chosen in the fitting, $\delta_{\mathrm{j}}=\omega_{\mathrm{j}}-$ $\omega_{\text {ref }}(j=1-4)$ with $\omega_{\text {ref }}$ being the resonance frequency of the reference system $\left(0.2 \mathrm{M} \mathrm{LiCl}\right.$ solution in $\left.\mathrm{D}_{2} \mathrm{O}\right)$. Among the four species, two species were assigned to have the same $\omega_{\mathrm{j}}$ value (the middle $\delta_{\mathrm{j}}$ value) because $H_{\text {high- } \delta}: H_{\text {middle- }-\delta}: H_{\text {low- } \delta} \cong 1: 2: 1$ at low $T$.

In Figures 5-7, the thick dotted curves indicate the best-fit line shape (real part of $g(\omega)$ with $\omega=\delta+\omega_{\text {ref }}$ ) for $N_{\mathrm{sp}}=4$, with $\kappa$ being utilized as the fitting parameter. Reasonable fitting is achieved under the above assumptions (equal-rate exchange among four species and $T, C, M$-insensitivity of $\omega_{\mathrm{j}}$ ), although some differences seen between the data and fitted curves in the low- $\delta$ regime may result from a limitation of these assumptions. (This limitation seems to mainly reflect some $T$-dependent changes in the chemical states of the aggregated $\mathrm{Li}$ species showing the resonance at low $\delta$.)

In Figure 9, the average exchange time (lifetime) of the Li species obtained from this fit, $\tau_{\mathrm{ex}}=1 / \kappa$, is plotted against $10^{3} T^{-1}$ ( $T=$ absolute temperature); see large symbols. The small symbols indicate a diffusion time $\tau_{\text {dif }}$ explained later. The plots of $\tau_{\mathrm{ex}}$ are not shown in the fast limit at high $T\left(\geq 323 \mathrm{~K}=50^{\circ} \mathrm{C}\right)$ where the exchange was rapid (as noted from the single NMR peak in Figures 5-7) and the spectra data could be fitted by eqs 7 and 8 with arbitrarily large $\kappa$ values. Similarly, some $\tau_{\mathrm{ex}}$ data at low $T$ are not shown because the corresponding ${ }^{7} \mathrm{Li}-\mathrm{NMR}$ spectra are in the slow limit and do not allow us to determine the accurate $\kappa$ values.

Figure 9 demonstrates that the $\tau_{\mathrm{ex}}$ data exhibit the Arrhenius behavior within the scatter of the plots
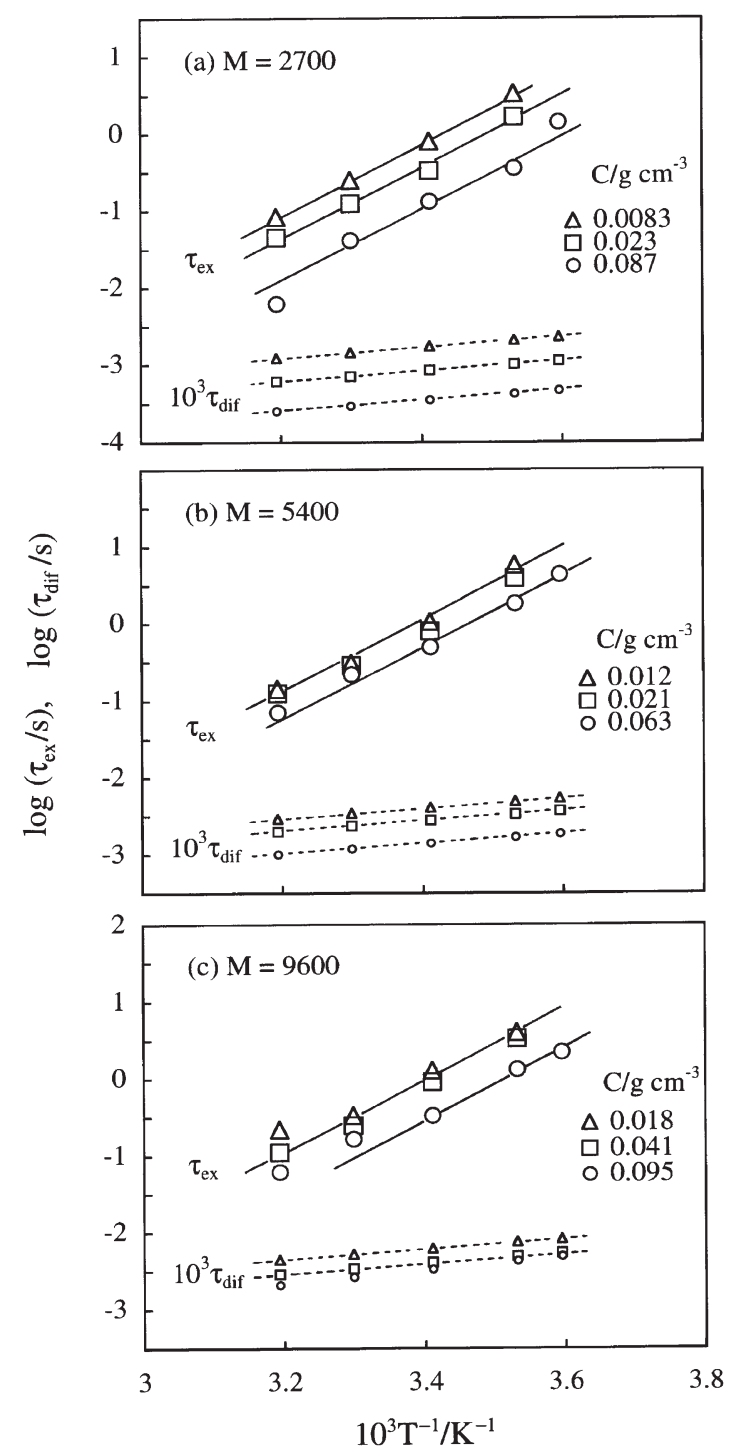

Figure 9. Temperature dependence of the $\mathrm{Li}-\mathrm{Li}$ exchange time $\tau_{\mathrm{ex}}$ obtained from ${ }^{7} \mathrm{Li}-\mathrm{NMR}$ measurements for the living PB chains with $M$ and $C$ as indicated (large symbols). Small symbols denote the time $\tau_{\text {dif }}$ required for the tetrameric aggregates of the chains to diffuse over the nearest neighbor distance. This $\tau_{\text {dif }}$ was estimated from the viscosity data.

and the activation energy hardly changes with $C$ and $M$. We also note that $\tau_{\text {ex }}$ decreases with increasing $C$ and decreasing $M$. These $C$ and $M$ dependencies, already noted for the raw spectrum (the spectrum at respective $T$ shows a faster-exchange pattern for larger $C$ and smaller $M$; $c f$. Figures 5-7), are most clearly demonstrated in Figure 10 where $\tau_{\mathrm{ex}}$ for the PB-3, PB-5, and PB-10 living chains at a constant temperature, $10^{\circ} \mathrm{C}$, are plotted against $C$ of the chains.

If the exchange of the associated Li species occurs through their independent/spontaneous dissociation as illustrated in Figure 11a, $\tau_{\mathrm{ex}}$ would be independent of $C$ and $M$ (and determined by the bare activation energy of this spontaneous dissociation). However, this is not the case for the PBLi chains, as seen in 


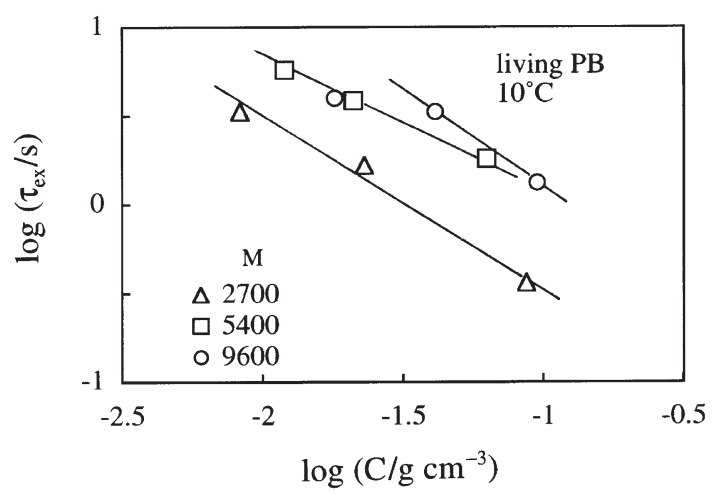

Figure 10. Concentration dependence of the $\mathrm{Li}-\mathrm{Li}$ exchange time $\tau_{\mathrm{ex}}$ for the living PB chains at $10^{\circ} \mathrm{C}$.

(a)

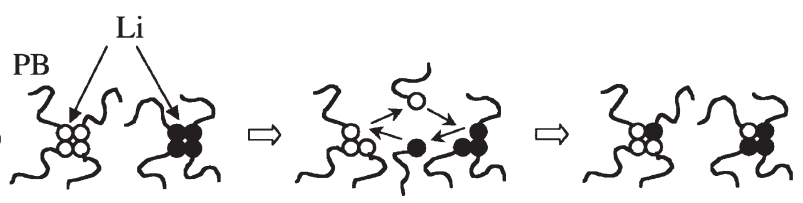

(b)
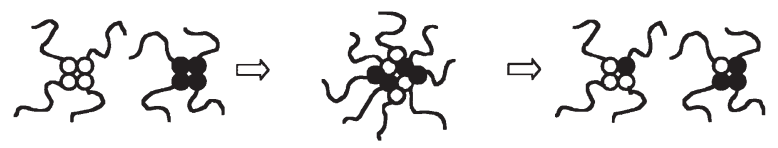

(c)

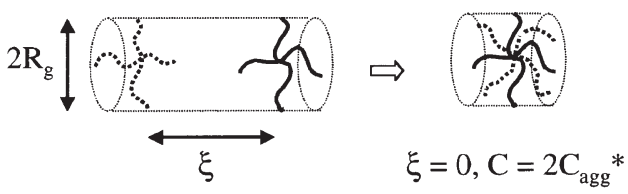

Figure 11. Schematic illustration for Li-Li exchange. Part (a) shows the non-cooperative (independent) exchange occurring through breakage of $\mathrm{Li}-\mathrm{Li}$ bonds and release of an isolated $\mathrm{Li}$ species. Part (b) shows the cooperative exchange occurring through formation of a transient, fused aggregate. Part (c) illustrates a geometry for calculating an increase of the osmotic free energy due to formation of the transient aggregate.

Figures 9 and 10. Thus, the exchange of the Li species should have occurred cooperatively, possibly through thermal collision of small star-like aggregates of the living PB chains containing the associated Li domains at their centers as illustrated in Figure 11b: This collision would allow a transient formation of a larger, fused aggregates of $\mathrm{Li}$, and these transient aggregates would split into smaller aggregates to allow the $\mathrm{Li}-\mathrm{Li}$ exchange without releasing the energetically unfavorable, isolated (non-aggregated) Li species. This mechanism of exchange is further discussed below in relation to the diffusive motion of the star-like aggregates of the PB chains.

\section{Thermal Collision and Dissociation of Aggregates}

The main component in the aggregates of the living PBLi chains is the tetrameric star-like aggregate, as discussed for Figure 4. The thermal collision rate of these aggregates, possibly affecting the $\mathrm{Li}-\mathrm{Li}$ exchange rate as discussed above, can be estimated from their viscosity. This viscosity cannot be straightforwardly estimated from the $\eta_{\mathrm{PB}}$ data of the living solutions, because of the non-negligible contribution of the coexisting large aggregates to those data; $c f$. bottom panels of Figures 2 and 3. In contrast, the deactivated $\mathrm{PB}$ chains behave as the moderately dilute chains to exhibit the Rouse-like $\eta_{\mathrm{PB}}$ data; $c f$. top panels of Figures 2 and 3. Since the star-like aggregates should also behave as the moderately dilute chains (as discussed earlier in relation to their $C_{\text {agg }}{ }^{*}$ ), their diffusivity and thermal collision rate can be estimated from the $\eta_{\mathrm{PB}}$ data of the deactivated PB chains on the basis of the Rouse-Ham relationship. ${ }^{26}$ We made this estimation in the following way.

For the $f$-arm star and linear chains at the same $C$, the latter being identical to the star arm, the diffusion coefficients $D$ in the Rouse-Ham regime satisfy a relationship, ${ }^{26} D_{\text {star }} / D_{\text {linear }}=1 / f$. Since $D_{\text {linear }}$ is related to the viscosity $\eta_{\text {linear }}$, radius of gyration $R_{\mathrm{g}, \theta}$, and molecular weight $M$ of the linear chain as $D_{\text {linear }}=$ $C_{R T R_{\mathrm{g}, \theta^{2}} / 6 \mathrm{M} \eta_{\text {linear }} \text { (Rouse relationship }}{ }^{26,31}$ ), we find

$$
D_{\text {star }}=\left(\frac{C R T}{6 f}\right)\left(\frac{R_{\mathrm{g}, \vartheta}^{2}}{M}\right)_{\text {linear }} \frac{1}{\eta_{\text {linear }}}
$$

An average distance between the neighboring $f$-mer aggregates is given by $\xi \cong\left(f M / C N_{\mathrm{A}}\right)^{1 / 3}$ with $N_{\mathrm{A}}$ being the Avogadro number. Thus, we obtain an estimate of a time $\tau_{\text {dif }}$ required for diffusion of the aggregate over this distance,

$$
\tau_{\text {dif }}=\frac{\xi^{2}}{6 D_{\text {star }}}=F \frac{M^{5 / 3} \eta_{\text {linear }}}{C^{5 / 3} R T R_{\mathrm{g}, \vartheta}^{2}} \text { with } F=\frac{f^{5 / 3}}{N_{\mathrm{A}}^{2 / 3}}
$$

The reciprocal of this time, $1 / \tau_{\mathrm{dif}}$, can be utilized as a measure of the thermal collision frequency for the star chains. The Li-Li exchange (through formation of the fused aggregate; Figure 11b) does not occur at this frequency if the exchange is associated with a large activation barrier, as discussed later in more details.

Utilizing the empirical equation for $R_{\mathrm{g}, \theta}$ (eq 2) and the $\eta_{\mathrm{PB}}$ data of the deactivated linear PB chains (Figure 1) in eq 10, we obtained $\tau_{\text {dif }}$ of the star-like tetrameric aggregates of the living $\mathrm{PB}$ chains $(f=4)$ at $C$ and $T$ examined in the viscosity experiments. Then, making short interpolation and/or extrapolation of these $\tau_{\text {dif }}$ values, we evaluated $\tau_{\text {dif }}$ at $C$ and $T$ examined in the ${ }^{7} \mathrm{Li}$-NMR measurements. In Figure 9, the $\tau_{\text {dif }}$ thus evaluated is multiplied by a factor of $10^{3}$ and plotted against $10^{3} T^{-1}$; see small symbols connected with the dotted lines. Clearly, $\tau_{\mathrm{dif}}$ is orders of magnitude shorter than the NMR-determined $\mathrm{Li}-\mathrm{Li}$ exchange time, $\tau_{\text {ex }}$ (large symbols). 
The Zimm relationship for infinitely dilute chains, ${ }^{26}$ $\eta_{\mathrm{PB}} \propto M^{1 / 2}$, was not observed for the deactivated PB chains and aggregates ( $c f$. Figure 3). However, for completeness, we also evaluated $\tau_{\text {dif }}{ }^{[\mathrm{Z}]}$ of the aggregates on the basis of the Zimm-Kilb dynamics. This $\tau_{\text {dif }}{ }^{[Z]}$ is given by eq 10 with $F=f^{2 / 3}\{(\sqrt{2}-1)+$ $\left.(2-\sqrt{2}) f^{-1}\right\}^{-1} N_{\mathrm{A}}{ }^{1 / 3} / 6.912 \Phi_{0} \quad\left(k_{\mathrm{B}}=\right.$ Boltzmann constant and $\Phi_{0}=$ Flory constant). ${ }^{26}$ Specicically, $\tau_{\text {dif }}{ }^{[Z]} \cong 0.14 \tau_{\text {dif }}$ for $f=4$, with $\tau_{\text {dif }}$ being the RouseHam diffusion time plotted in Figure 9 (small symbols). Thus, we can conclude that the Li-Li exchange is much slower than the thermal collision of the aggregates $\left(\tau_{\mathrm{ex}} \gg \tau_{\mathrm{dif}}{ }^{[\mathrm{Z}]}\right)$ even if the aggregates obey the Zimm-Kilb dynamics.

The above result has several important consequences. First of all, the Rouse-Ham type viscoelastic relaxation of the PBLi chains in the form of the star-like aggregates occurs in a time scale of $\tau_{\mathrm{r}}{ }^{\text {[star] }}=$ $24 M \eta_{\text {linear }} / \pi^{2} C R T=\left\{24 N_{\mathrm{A}}{ }^{2 / 3} C^{2 / 3} R_{\mathrm{g}, \theta^{2}} / \pi^{2} f^{5 / 3} M^{2 / 3}\right\}-$ $\tau_{\mathrm{dif}} \leq 0.2 \tau_{\mathrm{dif}}$ in the ranges of $C$ and $M$ examined. Thus, the Li-Li exchange (requiring the time $\tau_{\text {ex }} \gg$ $\tau_{\text {dif }}$ ) does not occur during the relaxation process of the aggregates, meaning that the viscosity of the living PB chains is determined by the thermal motion of their star-like aggregates. This result lends support to our analysis of $\tau_{\text {dif }}$ based on the Rouse-Ham dynamics. Note also that the ${ }^{7} \mathrm{Li}-\mathrm{NMR}$ spectra (Figures 57) significantly change with $T$ because the spectra reflect the $T$-sensitive exchange time $\tau_{\mathrm{ex}}$ (Figure 9) while the viscosity of the PBLi solutions (Figure 1) changes with $T$ only moderately because the viscosity is determined by the aggregate motion much faster than the exchange.

The second consequence is related to the thermal collision of the star-like aggregates of the PBLi chains. This collision (requiring the time $\tau_{\text {dif }}$ ) occurs for many times before the dissociation occurs through the $\mathrm{Li}-\mathrm{Li}$ exchange (in the time scale of $\tau_{\mathrm{ex}}$ ). Thus, we expect that the Li-Li exchange occurs cooperatively through collision-activated fusion of the small aggregates into a larger, transient aggregate (Figure 11b) and this fusion occurs only when the collision energy is sufficiently large (i.e., at a frequency $\ll$ collision frequency $\left.1 / \tau_{\mathrm{dif}}\right)$. For this cooperative exchange, the $\tau_{\mathrm{ex}}$ is proportional to $\tau_{\mathrm{dif}}$ (i.e., $\tau_{\mathrm{dif}}$ serves as a basic time-constant for the exchange) but a $\tau_{\mathrm{ex}} / \tau_{\mathrm{dif}}$ ratio is much larger than unity because of an activation barrier for the formation of the transient aggregates. In fact, the living PBLi chains have $\tau_{\mathrm{ex}} / \tau_{\mathrm{dif}} \gg 1$. Furthermore, the $\tau_{\mathrm{ex}} / \tau_{\text {dif }}$ ratio is not the same for the chains of various $C$ and $M$, as demonstrated in Figure 12a: The $\tau_{\mathrm{ex}} / \tau_{\text {dif }}$ ratio is considerably smaller for the PB-10 chain at $C=0.095 \mathrm{~g} \mathrm{~cm}^{-3}$ (filled circles) than for the same chains at lower $C$ (unfilled circles) and the other chains (triangles and squares). This difference of the
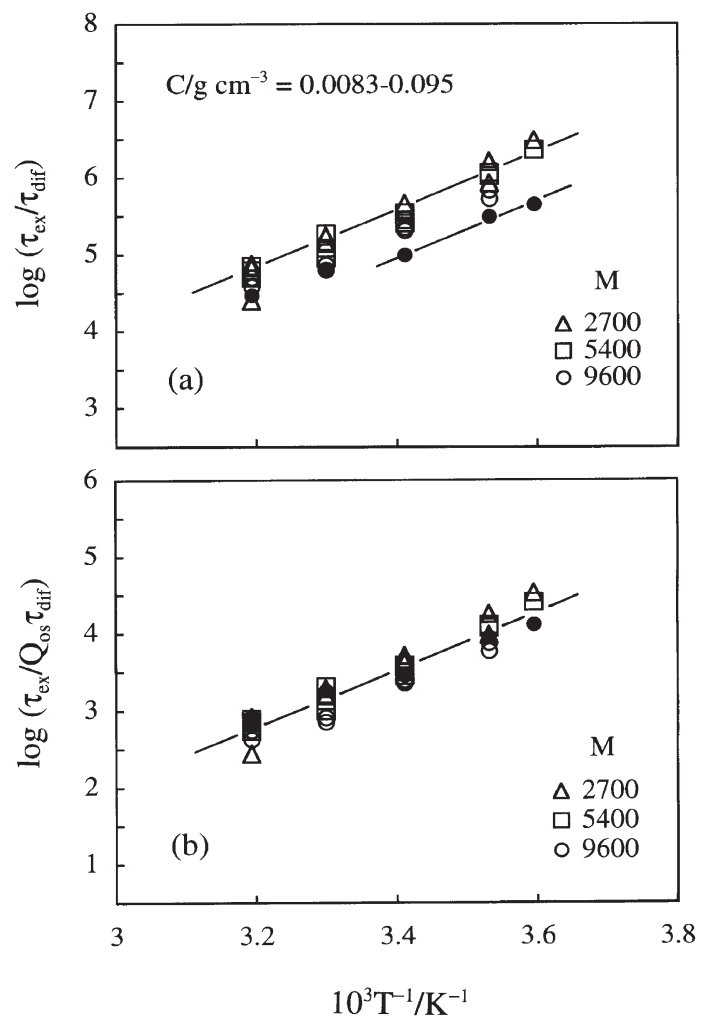

Figure 12. Temperature dependence of two kinds of reduced $\mathrm{Li}-\mathrm{Li}$ exchange time evaluated for the living PB chains, (a) $\tau_{\mathrm{ex}} /$ $\tau_{\text {dif }}$ and (b) $\tau_{\text {ex }} / Q_{\text {os }} \tau_{\text {dif }}$. The data for the chain with $M=9600$ at the highest $C\left(=0.095 \mathrm{~g} \mathrm{~cm}^{-3}\right)$ are indicated with filled circles, and the data for this chain at lower $C$ are denoted with unfilled circles. The unfilled triangles and squares show the data for the chains with $M=2700$ and 5400 , respectively.

$\tau_{\mathrm{ex}} / \tau_{\mathrm{dif}}$ ratio can be related to the activation barrier.

In the simplest activation process, we expect that two star-like (tetrameric) aggregates are compressed with each other to form a larger, transient aggregate, as illustrated in Figure 11b. This compressing process is accompanied by an increase of the osmotic free energy (osmotic penalty) $\Delta G_{\mathrm{os}}$ due to an increase of the local concentration. In the solution with $C<C_{\mathrm{agg}}{ }^{*}$, the aggregates are not overlapping on average before the compression and $\Delta G_{\mathrm{os}}$ would not increase significantly until the center-to-center distance between the aggregates $\xi$ is decreased below twice of their radius of gyration $R_{\mathrm{g}}$ (i.e., until the aggregates begin to overlap); $c f$. Figure 11c. In the solution with $C \geq C_{\text {agg }}{ }^{*}$, the aggregates are already overlapping at the onset of the compression and $\Delta G_{\mathrm{OS}}$ would increase on any decrease of $\xi$ from its onset value, $\left\{f M / C N_{\mathrm{A}}\right\}^{1 / 3}$ with $f M$ being the molecular weight of the aggregate. Thus, $\Delta G_{\mathrm{os}}$ can be estimated as

$$
\Delta G_{\mathrm{os}}=-\pi R_{\mathrm{g}}{ }^{2} \int_{\xi_{\text {start }}}^{0} \Pi \mathrm{d} \xi
$$

Here, $\xi_{\text {start }}$ is the distance where $\Delta G_{\mathrm{OS}}$ begins to increase significantly, $\xi_{\text {start }}=2 R_{\mathrm{g}}$ and $\left\{f M / C N_{\mathrm{A}}\right\}^{1 / 3}$ 
for the solutions with $C<C_{\mathrm{agg}}{ }^{*}$ and $C \geq C_{\mathrm{agg}}{ }^{*}$, respectively, $\Pi$ is the osmotic pressure, and the front factor $\pi R_{\mathrm{g}}{ }^{2}$ is the cross sectional area depicted in Figure 11c.

The calculation of $\Delta G_{\mathrm{os}}$ (eq 11) requires the $\Pi$ data for the overlapping aggregates. However, no osmotic data are available in literature for low- $M$ PB star chains in $\mathrm{Bz}$ similar to our star-like aggregates. For this reason, we assumed a scaling form of $\Pi$ known to be valid for high- $M$ star/linear chains in the semidilute regime: ${ }^{32,33}$

$$
\frac{f M \Pi}{C R T}=K_{\Pi}\left(\frac{C}{C_{\mathrm{agg}}{ }^{*}}\right)^{1 /(3 v-1)}
$$

Here, $K_{\Pi}$ is a constant of the order of unity, and $v$ is the molecular weight exponent for $R_{\mathrm{g}}\left(R_{\mathrm{g}} \propto M^{v}\right)$. During the compression process depicted in Figure 11c, the local concentration is related to the center-tocenter distance $\xi$ as $C=\beta\left(2 f M / N_{\mathrm{A}}\right) / \pi R_{\mathrm{g}}{ }^{2}\left(\xi+2 R_{\mathrm{g}}\right)$ where $\beta(=3 / 2)$ is a correction factor equating $C$ at $\xi=2 R_{\mathrm{g}}$ (onset of overlapping of the aggregates) and $C_{\mathrm{agg}}{ }^{*}=\left(f M / N_{\mathrm{A}}\right) /\left(4 \pi R_{\mathrm{g}}{ }^{3} / 3\right)$. From eqs 11 and 12 together with this relationship, $\Delta G_{\text {os }}$ is calculated to be

$$
\begin{aligned}
\Delta G_{\mathrm{os}}= & 3(3 v-1) k_{\mathrm{B}} T K_{\Pi} \\
\times & {\left[2^{1 /(3 v-1)}-\left(\frac{4 R_{\mathrm{g}}}{\xi_{\text {start }}+2 R_{\mathrm{g}}}\right)^{1 /(3 v-1)}\right] }
\end{aligned}
$$

The activation factor for this osmotic penalty, $Q_{\mathrm{os}}=$ $\exp \left(\Delta G_{\mathrm{os}} / k_{\mathrm{B}} T\right)$, is independent of $T$.

In the evaluation of $Q_{\text {os }}$, we chose $K_{\Pi}=1$ for simplicity and utilized $v=1 / 2$ because the excluded volume effect can be safely neglected for the aggregates of low- $M$ PB chains. Correspondingly, $R_{\mathrm{g}}$ of the tetrameric aggregates $(f=4)$ was evaluated from the Gaussian relationship in the absence of this effect, ${ }^{26}$ $R_{\mathrm{g}}=(3 f-2)^{1 / 2} f^{-1 / 2} R_{\mathrm{g}, \text { lin }}$ with $R_{\mathrm{g}, \text { lin }}$ being the radius of gyration of the deactivated linear PB chains given by eq 2 . In Figure $12 \mathrm{~b}$, the $\mathrm{Li}-\mathrm{Li}$ exchange time $\tau_{\text {ex }}$ is reduced by the diffusion time $\tau_{\mathrm{dif}}$ and the osmotic factor $Q_{\mathrm{os}}$. This reduced quantity is almost universally dependent on $T$ irrespective of $C$ and $M$ of the living PB chains. (We also utilized a virial expansion form ${ }^{26}$ of $\Pi$ to evaluate $Q_{\mathrm{os}}$. The resulting $Q_{\mathrm{os}}$ values were not significantly different from those obtained from the scaling form of $\Pi$, and the universality seen in Figure $12 \mathrm{~b}$ was not affected by our choice of the expression of $\Pi$.)

The above universal behavior is successfully cast in an Arrhenius-type empirical equation shown with the solid line in Figure 12b,

$$
\tau_{\text {ex }} \propto Q_{\text {os }} \tau_{\text {dif }} \exp (\Delta E / R T) \text { with } \Delta E \cong 88 \mathrm{~kJ} / \mathrm{mol}
$$

The activation energy $\Delta E$ is considerably smaller than the bare binding energy calculated with quantum chemical methods, ${ }^{34} \Delta E^{(\text {bare })} \cong 150 \mathrm{~kJ} / \mathrm{mol}$ for a tetramer of allyllithium. This difference seems to be a natural consequence of the cooperativity of the $\mathrm{Li}-\mathrm{Li}$ exchange process: The exchange would occur through formation of the transient aggregates (Figure 11b) without releasing an isolated $\mathrm{Li}$ species. Thus, the net activation energy $\Delta E$ for the exchange process is smaller than $\Delta E^{\text {(bare) }}$ required for breaking the Li$\mathrm{Li}$ bonds and releasing the isolated $\mathrm{Li}$ species.

In summary, the above results strongly suggest that the dissociation of the star-like, tetrameric aggregates of the PBLi chains requires the thermal collision of the aggregates under the osmotic barrier and that the exchange of the Li species allowing the dissociation is achieved cooperatively through formation of the transient, fused aggregate. Both of the thermal collision rate and osmotic barrier, represented by the $\tau_{\text {dif }}$ and $Q_{\text {os }}$ terms in eq 14, reflect the polymeric character of the PBLi chains. In other words, the dissociation kinetics of the aggregates is determined by not only the neat Li chemistry (reflected in $\Delta E$ ) but also the polymeric character of the constituent chains.

\section{CONCLUDING REMARKS}

We have examined the dynamic behavior and dissociation kinetics of the aggregates of living PBLi chains in d-Bz. The chains formed tetrameric aggregates as the main component, as confirmed from the scattering experiments. The diffusion time of these aggregates $\tau_{\text {dif }}$ estimated from the viscosity data was much shorter than the exchange time of the $\mathrm{Li}$ species $\tau_{\mathrm{ex}}$ (= lifetime of the associated Li domain) determined from ${ }^{7} \mathrm{Li}-\mathrm{NMR}$. The $\tau_{\mathrm{ex}}$ data were satisfactorily described by an empirical equation, $\tau_{\mathrm{ex}} \propto$ $Q_{\text {os }} \tau_{\text {dif }} \exp (\Delta E / R T)$, where $Q_{\text {os }}$ denotes an osmotic barrier for mutual approach of the aggregates of the living PB chains carrying the associated $\mathrm{Li}$ domains at the center. The activation energy $\Delta E(\cong 88 \mathrm{~kJ} / \mathrm{mol})$ was considerably smaller than the bare energy required for breaking the $\mathrm{Li}-\mathrm{Li}$ bonds and releasing isolated $\mathrm{Li}$ species. These results suggest that the collision of the aggregates (under the osmotic barrier) is required for the dissociation of the aggregates and the dissociation results from a cooperative exchange of $\mathrm{Li}$ species occurring through formation of a transient, fused aggregate. Thus, the dissociation kinetics of the aggregates is determined by not only the neat $\mathrm{Li}$ chemistry (reflected in $\Delta E$ ) but also the polymeric character of the constituent chains (reflected in $\tau_{\text {dif }}$ and $Q_{\mathrm{os}}$ ). 


\section{REFERENCES}

1. M. Morton, “Anionic Polymerization: Principles and Practice," Academic Press, New York, 1983.

2. M. Szwarc, Adv. Polym. Sci., 49, 1 (1983).

3. M. Szwarc and M. van Beylen, "Ionic Polymerization and Living Polymers," Chapman and Hall, New York, 1993.

4. D. J. Worsfold and S. Bywater, Macromolecules, 5, 393 (1972).

5. A. Duda and S. Penczek, Macromolecules, 27, 4876 (1994).

6. S. Bywater, Macromolecules, 27, 6221 (1994).

7. S. Bywater, Macromolecules, 31, 6010 (1998).

8. L. J. Fetters, N. P. Balsara, J. S. Huang, H. S. Jeon, K. Almdal, and M. Y. Lin, Macromolecules, 28, 4996 (1995).

9. J. Stellbrink, L. Willner, O. Jucknischke, D. Richter, P. Lindner, L. J. Fetters, and J. S. Huang, Macromolecules, 31, 4189 (1998).

10. J. Stellbrink, L. Willner, D. Richter, P. Linder, L. J. Fetters, and J. S. Huang, Macromolecules, 32, 5321 (1999).

11. M. Morton and L. J. Fetters, J. Polym. Sci., Part A: Polym. Chem., 2, 3331 (1964).

12. M. Morton, L. J. Fetters, and E. E. Bostick, J. Polym. Sci., Part C: Polym. Lett., 1, 311 (1963).

13. M. Morton, L. J. Fetters, R. A. Pett, and J. F. Meier, Macromolecules, 3, 3273 (1970).

14. M. M. Al-Jarrah and R. N. Young, Polymer, 21, 119 (1980).

15. M. Szwarc and H. C. Wang, Macromolecules, 15, 208 (1982).

16. A. Z. Niu, J. Stellbrink, J. Allgaier, L. Willner, D. Richter, B. W. Koenig, M. Gondorf, S. Willbold, L. J. Fetters, and R. P. May, Macromol. Symp., 215, 1 (2004).

17. A. Z. Niu, J. Stellbrink, J. Allgaier, L. Willner, A. Radulescu, D. Richter, B. W. Koenig, R. P. May, and L. J.
Fetters, J. Chem. Phys., 122, 134906 (2005).

18. Y. Matsuda, T. Sato, Y. Oishi, and H. Watanabe, J. Polym. Sci., Part B: Polym. Phys., 43, 1401 (2005).

19. H. Watanabe, Y. Oishi, T. Kanaya, H. Kaji, and F. Horii, Macromolecules, 36, 220 (2003).

20. H. Watanabe, O. Urakawa, H. Yamada, and M. L. Yao, Macromolecules, 29, 755 (1996).

21. "Kagaku Binran II (Chemistry Handbook)," 3rd ed, The Chemical Society Japan, Ed., Maruzen, Tokyo, 1984.

22. E. D. Becker, "High Resolution NMR, Theory and Chemical Applications," 2nd ed, Academic Press, New York, 1980.

23. A. Abragam, "The Principles of Nuclear Magnetism," Oxford Press, Clarendon, 1989.

24. L. J. Fetters, N. Hadjichristidis, J. S. Linder, and J. M. Mays, J. Phys. Chem. Ref. Data, 23, 619 (1994).

25. J. D. Ferry, "Viscoelastic Properties of Polymers," 3rd ed, Wiley, New York, 1980.

26. H. Yamakawa, "Modern Theory of Polymer Solutions," Harper \& Row, New York, 1971.

27. G. Beaucage and D. Schaefer, J. Non-Cryst. Solids, 172, 797 (1994).

28. H. Benoit, J. Polym. Sci., 11, 507 (1953).

29. K. Huber, S. Buntle, P. Lutz, and W. Burchard, Macromolecules, 18, 1461 (1985).

30. M. Mehring, "High-Resolution NMR in Solids," Springer, Berlin, 1983.

31. M. Doi and S. F. Edwards, "The Theory of Polymer Dynamics," Oxford Press, Clarendon, 1986.

32. Y. Higo, N. Ueno, and I. Noda, Polym. J., 15, 367 (1983).

33. I. Noda, Y. Higo, N. Ueno, and T. Fujimoto, Macromolecules, 17, 1055 (1984).

34. A. Frischknecht and S. T. Milner, J. Chem. Phys., 114, 1032 (2001). 